Research Article

\title{
Annihilators in Universal Algebras: A New Approach
}

\author{
Gezahagne Mulat Addis $(D)$ \\ Department of Mathematics, University of Gondar, Gondar, Ethiopia \\ Correspondence should be addressed to Gezahagne Mulat Addis; buttu412@yahoo.com
}

Received 8 March 2020; Accepted 29 June 2020; Published 20 July 2020

Academic Editor: Andrei V. Kelarev

Copyright ( 2020 Gezahagne Mulat Addis. This is an open access article distributed under the Creative Commons Attribution License, which permits unrestricted use, distribution, and reproduction in any medium, provided the original work is properly cited.

The purpose of this paper is to study annihilators and annihilator ideals in a more general context; in universal algebras.

\section{Introduction}

Annihilators and annihilator ideals have been studied in different classes of algebras. In ring theory, Bear rings, quasiBear rings, and principally quasi-Baer rings are defined using left and right annihilator ideals (see [1-6]). The notion of annihilators is also closely connected to that of regular rings, specifically to biregular rings, Von Neumann regular rings, self-injective rings, and continuous regular rings (see [7-12]). More recently, annihilators were studied in local cohomology modules (see [13-16]).

On the contrary, the concept of annihilators in lattices was studied by Mandelker [17] and later extended to the class of distributive lattices by Cornish [18], Speed [19], and Davey [20]. In [21], Davey and Nieminen studied the structure of annihilators in modular lattices. Halaš [22] studied annihilators in ordered sets as a generalization of lattices. There are also other classes of algebras for which annihilators and annihilator ideals have a great deal of applications. For instance, a class of BCK-algebras [23], Banach algebras [24], standard QBCC algebras [25], weakly standard BCC-algebras [26], C-algebras [27, 28], and others.

In recent times, the theory of ideals has been taking place in a more general context. Gumm and Ursini [29] introduced the concept of ideals in universal algebras having a constant 0 as a generalization of those familiar structures: normal subgroups (in groups), ideals (in rings), submodules (in modules), subspaces (in vector spaces), and filters (in implication algebras or Heyting algebras). They have also defined and characterized the commutator (or the product) of ideals in universal algebras. Later, Ursini [30] applied this product to define and study prime ideals in universal algebras. He has also defined relative annihilator of ideals under a name "residual of ideals."

The general concept of annihilators in universal algebras was introduced by Chajda and Halas in the paper [31] based on annihilator terms. In the present paper, we propose another approach to study annihilators and annihilator ideals in universal algebras by the use of the commutator of ideals in the sense of Gumm and Ursini [29]. We define $c$-unit elements in an algebra $A$ and obtain some of their properties. By applying the concept of relative annihilators, it is shown that the class of ideals of an algebra $A$ with a $c$-unit forms a complete residuated lattice. We also obtain a class of algebras called $c$-idempotent algebras, for which the lattice of all annihilator ideals of each algebra forms a Boolean algebra. This class of algebras contains well-known structures, lattices with least element $0, C$-algebras, Boolean rings, and regular rings. It is observed that the commutator of ideals in $c$-idempotent algebras is the same as their intersection. Moreover, let every singleton set $\{a\}$ in an algebra $A$ be an $m$--system, which is a necessary and sufficient condition for $A$ to be $c$-idempotent. Furthermore, $c$-idempotent algebras satisfy an important property, known as the separation axiom.

\section{Preliminaries}

This section contains some definitions and results which will be used in this paper. Throughout this paper, $A \in \mathscr{K}$, where $\mathscr{K}$ is a class of algebras of a fixed type $\Omega$, and we assume that 
there is an equationaly definable constant in all algebras of $\mathscr{K}$ denoted by 0 . For a positive integer $n$, we write $\vec{a}$ to denote the $n$-tuple $\left\langle a_{1}, a_{2}, \ldots, a_{n}\right\rangle \in A^{n}$. For the standard concepts in universal algebras, we refer to [32-34].

Definition 1 (see [29]). A term $p(\vec{x}, \vec{y})$ is said to be an ideal term in $\vec{y}$ if

$$
p\left(a_{1}, \ldots, a_{n}, 0,0, \ldots, 0\right)=0
$$

for all $a_{1}, \ldots, a_{n} \in A$. A nonempty subset $I$ of $A$ will be called an ideal of $A$ if $p\left(a_{1}, \ldots, a_{n}, b_{1}, \ldots, b_{m}\right) \in I$ for all $a_{1}, \ldots, a_{n} \in A, b_{1}, \ldots, b_{m} \in I$ and any ideal term $p(\vec{x}, \vec{y})$ in $\vec{y}$.

We denote the class of all ideals of $A$ by $i(A)$. It is easy to check that the intersection of any family of ideas of $A$ is an ideal. So, for a subset $S \subseteq A$, always there exists a smallest ideal of $A$ containing $S$ which we call the ideal of $A$ generated by $S$ and it is denoted by $\langle S\rangle$. Note that $x \in\langle S\rangle$ if and only if $x=p\left(a_{1}, \ldots, a_{n}, b_{1}, \ldots, b_{m}\right)$ for some $a_{1}, \ldots, a_{n} \in A$, $b_{1}, \ldots, b_{m} \in S$, where $p(\vec{x}, \vec{y})$ is an $(n+m)$-ary ideal term in $\vec{y}$. If $S=\{a\}$, then we write $\langle a\rangle$ instead of $\langle S\rangle$. In this case, $x \in\langle a\rangle$ if and only if $x=p\left(a_{1}, \ldots, a_{n}, a\right)$ for some $a_{1}, \ldots, a_{n} \in A$, where $p(\vec{x}, \vec{y})$ is an $(n+1)$-ary ideal term in $\vec{y}$.

Definition 2 (see [30]). A nonzero element $u$ in $A$ is said to be a formal unit if $A=\langle u\rangle$, i.e., $A$ is generated by $u$ as an ideal.

Example 1. (1) If $G$ is a nontrivial cyclic group generated by the element $a$, then $a$ is a formal unit.

(2) If $R$ is a division ring, then every nonzero element in $R$ is a formal unit.

(3) The largest element in a bounded distributive lattice is a formal unit.

(4) Every maximal element in an almost distributive lattice is a formal unit.

Remark 1. A formal unit in an algebra (if it exists) is not necessarily unique (e.g., cyclic groups and almost distributive lattices may have several formal units).

Definition 3 (see [29]). A class $\mathscr{K}$ of algebras is called an ideal determined class if every ideal $I$ of $A \in \mathscr{K}$ is the zero congruence class of a unique congruence relation denoted by $I^{\delta}$. In this case, the map $I \mapsto I^{\delta}$ defines an isomorphism between the lattice of ideals and congruences on $A$.

Definition 4 (see $[29,30])$. A term $t(\vec{x}, \vec{y}, \vec{z})$ is said to be a commutator term in $\vec{y}, \vec{z}$ if it is an ideal term in $\vec{y}$ and an ideal term in $\vec{z}$.

An operation called the commutator (or the product) of ideals proposed by Gumm and Ursini [29] and later studied by Ursini [30] will be considered useful for our purposes.

Definition 5 (see [30]). For each $I, J \in I(A)$, their commutator $[I, J]$ is defined by

$$
\begin{aligned}
{[I, J]=} & \left\{t(\vec{a}, \vec{i}, \vec{j}): \vec{a} \in A^{n}, \vec{i} \in I^{m} \text { and } \vec{j} \in J^{k},\right. \\
& t(\vec{x}, \vec{y}, \vec{z}) \text { is a commutator term in } \vec{y}, \vec{z}\} .
\end{aligned}
$$

For subsets $H, G$ of $A,[H, G]$ denotes the product $[\langle H\rangle,\langle G\rangle]$. In particular, for $a, b \in A,[\langle a\rangle,\langle b\rangle]$ is denoted by $[a, b]$.

It is immediate from the definition that $[I, J]$ is an ideal of $A$ such that $[I, J] \subseteq I \cap J$. Moreover, one can observe that $[$,$] is commutative and increasing in both arguments. The$ following theorem gives a characterization for the commutator of ideals using the general commutator of congruences in ideal determined varieties. About the commutator of congruences see $[35,36]$.

Theorem 1 (see [30]). In an ideal determined variety, the commutator $[I, J]$ of ideals $I$ and $J$ is the zero congruence class of the commutator congruence $\left[I^{\delta}, J^{\delta}\right]$.

It is also observed that, in ideal determined varieties, the commutator of ideals is distributive over arbitrary joins of ideals. Throughout this paper $\mathscr{K}$ is assumed to be an ideal determined variety. Further detail on ideals (and their commutator) in universal algebras can be found in $[30,37-42]$.

Definition 6 (see [43]). An algebra $A$ is called idempotent if, for all $x \in A$,

$$
f(x, x, \ldots, x)=x,
$$

holds for each of its fundamental operations $f$.

It is evident that $A$ is idempotent if and only if every singleton set $\{x\}$ in $A$ is a subuniverse of $A$.

\section{Relative Annihilators}

In this section, we define relative annihilators in universal algebras and prove some of their basic properties. Mainly, we show that the class of ideals in an algebra $A$ forms a complete residuated lattice by the use of relative annihilators.

Definition 7. Let $I \in i(A)$. For any subset $S$ of $A$, we define

$$
(S: I)=\{a \in A:[a, S] \subseteq I\} .
$$

We call $(S: I)$, the annihilator of $S$ relative to $I$.

In the following theorem, we give another description for the relative annihilator.

Theorem 2. For any $S \subseteq A$ and an ideal $I$ of $A$,

$$
(S: I)=\cup\{H \in i(A):[H, S] \subseteq I\} \text {. }
$$

Proof. Let us define two sets $E$ and $G$ as follows:

$$
\begin{aligned}
& E=\bigcup\{H \in i(A):[H, S] \subseteq I\}, \\
& G=\{a \in A:[a, S] \subseteq I\} .
\end{aligned}
$$


We show that $E=G$. Let $a \in E$. Then, there is $H \in i(A)$ such that $a \in H$ and $[H, S] \subseteq I$. As $a \in H$, we have $\langle a\rangle \subseteq H$. This implies that $[a, S] \subseteq[H, S] \subseteq I$. So, $a \in G$ and hence $E \subseteq G$. Let $a \in G$. Then, $[a, S] \subseteq I$. If we take $H=\langle a\rangle$, then $H \in i(A)$ such that $a \in H$ and $[H, S] \subseteq I$ so that $a \in E$ and $G \subseteq E$. Therefore, $E=G$.

In the next theorem, we show that the annihilator of any subset $S$ relative to any ideal $I$ forms an ideal by applying the above theorem.

Theorem 3. For any $S \subseteq A$ and an ideal $I$ of $A,(S: I)$ is an ideal of $A$.

Proof. Clearly, $[0, S] \subseteq(0) \subseteq I$ so that $0 \in(S: I)$ and hence it is nonempty. Let $\vec{b}=\left\langle b_{1}, b_{2}, \ldots, b_{m}\right\rangle$ be an $m$-tuples of elements from $(S: I)$. Then, by Theorem 2, there exist $H_{1}, H_{2}, \ldots, H_{m} \in i(A)$ such that each $b_{i} \in H_{i}$ and $\left[H_{i}, S\right] \subseteq I$ for all $i$. If we put $H=\vee_{i=1}^{m} H_{i}$, then $H \in i(A)$ such that each $b_{i} \in H$ and $p(\vec{x}, \vec{y})$. If $\vec{a} \in A^{n}$ and $p(\vec{x}, \vec{y})$ is an ideal term in $\vec{y}$, then, as $H$ is an ideal, we get $p(\vec{a}, \vec{b}) \in H \subseteq(S: I)$. Therefore, $(S: I)$ is an ideal of $A$.

Theorem 4. For any subsets $S$ and $T$ of $A$ and ideals $I$ and $J$ of $A$, we have the following:

(1) $I \subseteq(S: I)$

(2) $[J, S] \subseteq I$ if and only if $J \subseteq(S: I)$

(3) $S \subseteq I \Longleftrightarrow(S: I)=A$

(4) $(I: J)$ is the largest ideal of A such that $[(I: J), I] \subseteq J$

(5) $S \subseteq T \Longrightarrow(T: I) \subseteq(S: I)$

(6) $I \subseteq J \Longrightarrow(S: I) \subseteq(S: J)$

(7) $(S: I \cap J)=(S: I) \cap(S: J)$

(8) $(I \vee J: K)=(I: K) \cap(J: K)$

Proof. Let $S$ and $T$ be subsets of $A$ and $I, J \in i(A)$ :

(1) Since $[I, S] \subseteq I$, then $I \subseteq(S: I)$.

(2) If $[J, S] \subseteq I$, then it is clear from the definition that $J \subseteq(S: I)$. Now suppose that $J \subseteq(S: I)$. For each $a \in J$, there exists $H_{a} \in i(A)$ such that $a \in H_{a}$ and $\left[H_{a}, S\right] \subseteq I$. Let us take $H=\vee_{a \in J} H_{a}$, such that $a \in H$ for all $a \in J$. Since the commutator [,] of ideals is distributive over arbitrary join, we get $[H, S] \subseteq I$. Now, consider

$$
[J, S] \subseteq[H, S] \subseteq I .
$$

Therefore, $[J, S] \subseteq I$.

(3) Suppose that $S \subseteq I$. Then, $\langle S\rangle \subseteq I$. For any $a \in A$, we have $[a, S] \subseteq\langle S\rangle \subseteq I$. Therefore, $(S: I)=A$.

(4) We first show that $[(I: J), I] \subseteq J$. Since $(I: J)$ is an ideal of $A$, it can be described as follows:

$$
(I: J)=\bigvee_{a \in(I: J)}\langle a\rangle \text {. }
$$

Also, we have $[a, I] \subseteq J$ for all $a \in(I: J)$. That is,

$$
\bigvee_{a \in(I: J)}[a, I] \subseteq J
$$

Since the commutator [,] of ideals is distributive over arbitrary join, it follows that $[(I: J), I] \subseteq J$. Now, let $K$ be any other ideal of $A$ such that $[K, I] \subseteq J$. Then, it is clear from the definition that $K \subseteq(I: J)$. Hence, the result holds.

(5) If $S \subseteq T$, then $[H, S] \subseteq[H, T]$ for all $H \in i(A)$. This confirms that $(T: I) \subseteq(S: I)$.

(6) Suppose that $I \subseteq J$. If $H$ is an ideal of $A$ such that $[H, S] \subseteq I$, then $[H, S] \subseteq J$. Therefore, $(S: I) \subseteq(S: J)$.

(7) It follows from (6) that $(S: I \cap J) \subseteq(S: I) \cap(S: J)$. If $a \in(S: I)$ and $a \in(S: J)$, then $[a, S] \subseteq I$ and $[a, S] \subseteq J$, that is, $[a, S] \subseteq I \cap J$ so that $a \in(S: I \cap J)$. Therefore, $(S: I) \cap(S: J) \subseteq(S: I \cap J)$ and the equality holds.

(8) It follows from (5) that $(I \vee J: K) \subseteq(I: K) \cap(J: K)$. If $a \in(I: K)$ and $a \in(J: K)$, then $[a, I] \subseteq K$ and $[a, J] \subseteq K \quad$ so that $[a, I \vee J]=[a, I] \vee[a, J] \subseteq K$. Therefore, $a \in(I \vee J: K)$ and hence the equality holds.

Theorem 5. If $\left\{J_{\alpha}\right\}_{\alpha \in \Delta}$ is a family of ideals of $A$ and I an ideal of $A$, then

$$
\left(\bigcup_{\alpha \in \Delta} J_{\alpha}: I\right)=\bigcap_{\alpha \in \Delta}\left(J_{\alpha}: I\right)
$$

Proof. The proof follows from (8) of Theorem 4, by applying the fact that the commutator [,] of ideals is distributive over arbitrary join.

Definition 8. A nonzero element $u \in A$ is a called a commutator unit (or $c$-unit for short) in $A$ if $[a, u]=\langle a\rangle$ for all $a \in A$.

\section{Example 2}

(1) In a ring $R$ with unity, the elements 1 and -1 are $c$-unit

(2) In a bounded distributive lattice $L$, the largest element 1 is $c$-unit

(3) In a bounded Hilbert algebra $A$, the least element 0 is c-unit

(4) Every maximal element in an almost distributive lattice is $c$-unit

Note also that every $c$-unit element in $A$ is a formal unit. This can be verified as follows: suppose that $u \in A$ is $c$-unit. Then, for each $a \in A$,

$$
a \in\langle a\rangle=[a, u] \subseteq[A, u] \subseteq\langle u\rangle .
$$

So that $A=\langle u\rangle$ and hence $u$ is a formal unit. However, the converse does not holds in general. This can be verified in the following example.

Example 3. Let $G$ be a nontrivial cyclic group generated by the element $a$. Then, $a$ is a formal unit but not a $c$-unit. 
Remark 2

(1) As can be seen from (1) of Example 2, c-unit (if it exists) need not necessarily be unique

(2) If $A$ has a $c$-unit $u$, then for each ideal $I$ of $A$, it holds that $[I, A]=I$

Let us now recall the definition of residuated lattices.

Definition 9. An algebra $\mathrm{L}=\langle L, \wedge, \vee, \otimes, \longrightarrow, 0,1\rangle$ of type $\langle 2,2,2,2,0,0\rangle$ is called a complete residuated lattice; the following conditions are satisfied:

(1) $\langle L, \wedge, \vee, 0,1\rangle$ is a complete lattice with the least element 0 and the greatest element 1 .

(2) $\langle L, \otimes, 1\rangle$ is a commutative monoid, i.e., $\otimes$ is commutative and associative and $a \otimes 1=a$ for each $a \in L$.

$(3) \otimes$ and $\longrightarrow$ satisfy the adjointness property, i.e.,

$$
a \otimes b \leq c, \quad \text { if and only if } a \leq b \longrightarrow c,
$$

for each $a, b, c \in L$ ( $\leq$ denotes the lattice ordering).

Theorem 6. Suppose that $c$ has c-unit elements. If the commutator [,] of ideals is associative, then $\langle i(A), \cap, \vee, \otimes, \longrightarrow,(0), A\rangle$ is a complete residuated lattice, where the binary operations $\otimes$ and $\longrightarrow$ on $i(A)$ are defined as follows. For each $I, J \in i(A)$,

$$
\begin{aligned}
I \otimes J & =[I, J], \\
I \longrightarrow J & =(I: J) .
\end{aligned}
$$

Proof. It is observed in [29] that the lattice $(i(A), \cap$, $\mathrm{V},(0), A)$ is a complete lattice withe least element $(0)$ and the largest element $A$. It is also clear from our assumption that $\langle i(A), \otimes, A\rangle$ is a commutative monoid. Moreover, it is observed in Theorem 4 (2) that $\otimes$ and $\longrightarrow$ satisfy adjointness property. Therefore, $\langle i(A), \cap, \vee, \otimes, \longrightarrow,(0), A\rangle$ is a complete residuated lattice.

Corollary 1. If the commutator of ideals in A coincides with their intersection, then $i(A)$ forms a Heyting algebra, where $\longrightarrow$ is as given in the previous theorem.

Distributive lattices with least element $0, c$-algebras, and almost distributive lattices are examples of algebras in which the commutator is the same as the set theoretic intersection, and hence their lattice of ideals forms a Heyting algebra.

An element $a$ in a ring $R$ is said to be idempotent if $a^{2}=a$. By imitating this property to the general case of universal algebras, we define the following.

Definition 10. An element $a \in A$ is called idempotent if

$$
[a, a]=\langle a\rangle \text {. }
$$

If all elements of $A$ are idempotent, then we call $A$ a commutator idempotent algebra (or a $c$-idempotent algebra for short). In other words, the commutator of ideals in $A$ is idempotent.

Boolean rings and, more generally, lattices with least element 0 are the most natural examples of $c$-idempotent algebras. One can also verify that regular rings (a ring $R$ is regular if for each $x \in R$ there exists $a \in R$ such that xax $=x$ ) are examples of $c$-idempotent algebras.

Remark 3. The prefix " $c$-" standing for the word "commutator" is added to differentiate with those idempotent algebras defined as in Definition 6.

One of the most important property of $c$-idempotent algebras is that the commutator of ideals coincides with their intersection. The following definition is taken from [30].

Definition 11. A nonempty subset $M$ of $A$ is said to be an $m$-system if $M \cap[a, b] \neq \varnothing$ for all $a, b \in M$.

The following theorem gives a necessary and sufficient condition for an algebra $A$ to be $c$-idempotent.

Theorem 7. An algebra $A$ is c-idempotent if and only if every singleton set $\{a\}$ in $A$ is an $m$-system.

Theorem 8. (the separation axiom). Let $A$ be a c-idempotent algebra, $J \in i(A)$ and $a \in A$ such that $a \notin J$. Then, $J$ and $a$ can be separated by a prime ideal, i.e., there is a prime ideal $P$ of $A$ containing $J$ and not containing $a$.

Proof. Let us define a set:

$$
\mathscr{F}=\{H \in i(A): J \subseteq H, a \notin H\} .
$$

Then, $\mathscr{F}$ is nonempty, and it forms a poset under the usual inclusion order. Moreover, one can easily verify that $\mathscr{F}$ satisfies the hypothesis of Zorn's lemma so that we can choose a maximal element, say $M$ in $\mathscr{F}$. That is, $M \in i(A)$ such that $J \subseteq M$ and $a \notin M$. Now, it is enough to show that $M$ is prime, suppose not. Then, there exist $x, y \in A$ such that $[x, y] \subseteq M \quad$ but $\quad x, y \notin M$. Put $M_{1}=M \vee\langle x\rangle \quad$ and $M_{2}=M \vee\langle y\rangle$. Then, $M_{1}$ and $M_{2}$ are ideals of $A$ properly containing $M$. By the maximality of $M$ in $\mathscr{F}$, both $M_{1}$ and $M_{2}$ do not belong to $\mathscr{F}$. So, $a \in M_{1}$ and $a \in M_{2}$ which gives $[a, a] \subseteq\left[M_{1}, M_{2}\right] \subseteq M$. Since $A$ is a $c$-idempotent algebra, we get $a \in M$, which is a contradiction. Therefore, $M$ is a prime ideal of $A$ such that $J \subseteq M$ and $a \notin M$.

Theorem 9. For each $I, J \in i(A)$,

$$
(I: J) \subseteq \cap\{P: P \text { is a prime ideal of } A, J \subseteq P \text { and } I \nsubseteq P\} \text {. }
$$

Moreover, if $A$ is c-idempotent, then the equality holds.

Proof. Let $P$ be any prime ideal of $A$ such that $J \subseteq P$ and $I \nsubseteq P$. If $a \in(I: J)$, then $[a, I] \subseteq J \subseteq P$. So, $[a, I] \subseteq P$. Since $P$ is prime and $I \nsubseteq P$, we get $a \in P$. Therefore,

$$
(I: J) \subseteq \cap\{P: P \text { is a prime ideal of } A, J \subseteq P \text { and } I \nsubseteq P\} .
$$


To prove the other inclusion, assume that $A$ is $c$-idempotent and let $a \notin(I: J)$. Then, $[a, I] \nsubseteq J$. Let us choose $b \in[a, I]$ such that $b \notin J$. By the separation axiom, there exists a prime ideal $P$ of $A$ such that $J \subseteq P$ and $b \notin P$ which gives $[a, I] \nsubseteq P$. That is, $a \notin P$ and $I \nsubseteq P$. Hence,

$\cap\{P: P$ is a prime ideal of $A, J \subseteq P$ and $I \nsubseteq P\} \subseteq(I: J)$,

and therefore the equality holds.

Corollary 2. For every ideal $J$ of a c-idempotent algebra A, the following holds:

$$
J=\cap\{P: P \text { is a prime ideal of } A \text { such that } J \subseteq P\} .
$$

Proof. We first show that $(A: J)=J$. Clearly, $J \subseteq(A$ : $J)$. Let $x \in(A: J)$. Then, $[x, A] \subseteq J$. Since $\mathrm{A}$ is a $c$-idempotent algebra, it follows that $x \in\langle x\rangle=[x, x] \subseteq[x, A] \subseteq J$ and hence the equality holds. Thus, the result follows from Theorem 9.

\section{Annihilator Ideals}

In this section, we study annihilator ideals in universal algebras. We begin by defining the annihilator of subsets of $A$.

Definition 12. For a subset $S$ of $A$, the annihilator of $S$ denoted by $S^{*}$ is defined to be

$$
S^{*}=(S:(0)) \text {. }
$$

If $S=\{a\}$, then we denote $S^{*}$ by $(a)^{*}$.

It is proved in the previous section that $S^{*}$ is an ideal of $A$ and $S^{*}=\langle S\rangle^{*}$ for all $S \subseteq A$. Also, it can be verified that $(0)^{*}=A$ and if $A$ has a $c$-unit or $A$ is c-idempotent, then $A^{*}=(0)$. The following corollary gives a prime representation for annihilator of a set in $c$-idempotent algebras.

Corollary 3. For any subset $S$ of a c-idempotent algebra $A$,

$$
S^{*}=\bigcap\{P: P \text { is a prime ideal of } A \text { such that } S \nsubseteq P\} \text {. }
$$

Proof. The proof follows from Theorem 9.

Lemma 1. For any subsets $S$ and $T$ of $A$,
(1) $S^{*}=\bigcap_{a \in S}(a)^{*}$
(2) $S \subseteq S^{* *}$
(3) $S^{* * *}=S^{*}$
(4) $S \subseteq T \Longrightarrow T^{*} \subseteq S^{*}$
(5) $S \subseteq T \Longrightarrow S^{* *} \subseteq T^{* *}$
(6) $(S \cup T)^{*}=S^{*} \cap T^{*}$

Theorem 10. The following holds for all $I, J \in i(A)$ :

(1) $\left[I, I^{*}\right]=(0)$, and if $A$ is c-idempotent, then $I \cap I^{*}=(0)$

(2) $[I, J]=(0)$ if and only if $I \subseteq J^{*}$
(3) $(I \vee J)^{*}=I^{*} \cap J^{*}$

Theorem 11. Let A be a c-idempotent algebra. Then,

$$
(I \cap J)^{* *}=I^{* *} \cap J^{* *} .
$$

Proof. It is clear that $(I \cap J)^{* *} \subseteq I^{* *} \cap J^{* *}$, and we proceed to show the other inclusion. Let $a \in I^{* *}$ and $a \in J^{* *}$. Then, $\left[a, I^{*}\right]=(0)$ and $\left[b, J^{*}\right]=(0)$. That is, if $b \in A$ such that either $[b, I]=(0)$ or $[b, J]=(0)$, then $[a, b]=(0)$. Now, for any $b \in A$, consider the following:

$$
\begin{aligned}
b \in(I \cap J)^{*} & \Longrightarrow[b, I \cap J]=(0) \\
& \Longrightarrow[b,[I, J]]=(0) \\
& \Longrightarrow[b, I] \subseteq J^{*} \\
& \Longrightarrow[a,[b, I]]=(0) \\
& \Longrightarrow[a, b] \subseteq I^{*} \\
& \Longrightarrow[a,[a, b]]=(0) \\
& \Longrightarrow[[a, a], b]=(0) \\
& \Longrightarrow[a, b]=(0) \\
& \Longrightarrow a \in(I \cap J)^{* *} .
\end{aligned}
$$

Therefore, $I^{* *} \cap J^{* *} \subseteq(I \cap J)^{* *}$ and the equality holds.

Definition 13. An ideal $I$ of $A$ is called annihilator ideal if $I=S^{*}$ for some nonempty subset $S$ of $A$.

It is clear to see that (0) is an annihilator ideal. Moreover, if either $A$ has a $c$-unit or $A$ is $c$-idempotent, then it is an annihilator ideal. We denote by $i^{*}(A)$ the class of annihilator ideals of $A$.

Lemma 2. An ideal I of $A$ is an annihilator ideal if and only if $I^{* *}=I$.

Let us consider a map $\phi: i(A) \longrightarrow i(A)$ defined by

$$
\phi(I)=I^{* *},
$$

for all $I \in i(A)$. Then, the results in Lemma 1 confirm that $\phi$ is a closure operator on $i(A)$ and the closed elements with respect to $\phi$ are those annihilator ideals of $A$.

Theorem 12. Let $I, J \in i(A)$. Then, $I \sqcup J=\left(I^{*} \cap J^{*}\right)^{*}$ is the smallest annihilator ideal of $A$ containing both $I$ and $J$.

Proof. Clearly, $I \sqcup J$ is an annihilator ideal of $A$. Also, since $I^{*} \cap J^{*} \subseteq I^{*}$, we get $I \subseteq I^{* *} \subseteq\left(I^{*} \cap J^{*}\right)^{*}=I \sqcup J$. Similarly, $J \subseteq J^{* *} \subseteq\left(I^{*} \cap J^{*}\right)^{*}=I \sqcup J$. Now, let $K$ be any other annihilator ideal containing both $I$ and $J$. Then, $K^{*} \subseteq I^{*} \cap J^{*}$. So, $I \sqcup J=\left(I^{*} \cap J^{*}\right)^{*} \subseteq K^{* *}=K$ and this completes the proof.

Theorem 13. Let $A$ be a c-idempotent algebra. Then, $\left\langle i^{*}(A), \cap, \sqcup,{ }^{*},(0), A\right\rangle$ is a Boolean algebra.

Proof. It follows from Theorems 11 and 12 that $i^{*}(A)$ is closed under $\cap$ and $\sqcup$, respectively. Since $A$ is $c$-idempotent, the commutator of ideals coincides with their intersection. 
So, $\cap$ is distributive over the join $\vee$ of ideals. For any $I, J, K \in i^{*}(A)$, consider the following:

$$
\begin{aligned}
I \cap(J \sqcup K) & =I \cap\left(J^{*} \cap K^{*}\right)^{*} \\
& =I \cap(J \vee K)^{* *} \\
& =I^{* *} \cap(J \vee K)^{* *} \\
& =(I \cap(J \vee K))^{* *} \\
& =((I \cap J) \vee(I \cap K))^{* *} \\
& =\left((I \cap J)^{*} \cap(I \cap K)^{*}\right)^{*} \\
& =(I \cap J) \sqcup(I \cap K) .
\end{aligned}
$$

Therefore, $\left\langle i^{*}(A), \cap, \sqcup,(0), A\right\rangle$ is a bounded distributive lattice. Moreover, for each $I \in i^{*}(A), I \cap I^{*}=(0)$ and $I \sqcup I^{*}=\left(I^{*} \cap I^{* *}\right)^{*}=\left(I^{*} \cap I\right)^{*}=(0)^{*}=A$. That is, every element of $i^{*}(A)$ is complemented and hence it is a Boolean algebra.

Definition 14. An ideal $I$ of $A$ is called a dense ideal if $I^{*}=(0)$; otherwise, $I$ is called a nondense ideal.

If $A$ has a $c$-unit element, then it is a dense ideal. Moreover, every maximal ideal in $A$ is either dense or an annihilator ideal.

Theorem 14. If the commutator [,] of ideals is associative, then the class $\mathscr{D}$ of all dense ideals of $A$ is either empty or a dual-ideal (filter) of the lattice $i(A)$.

Proof. Suppose that $\mathscr{D}$ is nonempty. Let $I, J \in \mathscr{D}$. Then, $I^{*}=(0)=J^{*}$. For any $x \in A$, consider the following:

$$
\begin{aligned}
x \in(I \cap J)^{*} & \Longrightarrow[x, I \cap J]=(0) \\
& \Longrightarrow[x,[I, J]]=(0)(\text { since }[I, J] \subseteq I \cap J) \\
& \Longrightarrow[[x, I], J]=(0) \\
& \Longrightarrow[x, I] \subseteq J^{*}=(0) \\
& \Longrightarrow[x, I]=(0) \\
& \Longrightarrow x \in I^{*}=(0) \\
& \Longrightarrow x=0 .
\end{aligned}
$$

Thus, $I \cap J \in \mathscr{D}$. Also, let $I \in \mathscr{D}$ and $J \in i(A)$. Then, $I^{*}=$ (0) and hence we have $(I \vee J)^{*}=I^{*} \cap J^{*}=(0)$. Thus, $I \vee J \in \mathscr{D}$, and this completes the proof.

Lemma 3. Let $A$ be a c-idempotent algebra and $I \in i(A)$. Then,

$$
I^{*} \subseteq I \Longrightarrow I^{*}=(0)
$$

Proof. Suppose that $I^{*} \subseteq I$ and let $a \in I^{*}$. Then, $a \in I$ and $[a, a] \subseteq\left[I^{*}, I\right]=(0)$. Since $a \in[a, a]$, it holds $a=0$. Thus, $I^{*}=(0)$.

Theorem 15. Let $A$ be a c-idempotent algebra in which every prime ideal is nondense. Then, the following conditions hold:

(1) Every prime ideal is an annihilator ideal
(2) Every prime ideal is of the form $(a)^{*}$

(3) For all $I \in i(A), I^{*} \vee I^{* *}=A$

(4) For all $I, J \in i(A), I \cap J=(0)$ implies $I^{*} \vee J^{*}=A$

Proof. Let $P$ be a prime ideal of $A$.

(1) We show that $P=P^{* *}$. It is enough to show that $P^{* *} \subseteq P$. Let $a \in P^{* *}$. Then, $\left[a, P^{*}\right]=(0) \subseteq P$. Since $P$ is prime, either $a \in P$ or $P^{*} \subseteq P$. Since every prime ideal is nondense, it follows from Lemma 3 that $P^{*} \subseteq P$ is impossible. So, $a \in P$ and hence the equality holds. Therefore, $P$ is an annihilator ideal.

(2) Let $P$ be a prime ideal of $A$. Then, by our assumption, $P$ is nondense. There is a nonzero $a \in A$ such that $[a, P]=(0)$. Our claim is to show that $P=(a)^{*}$. By (1), we have $P=P^{* *}$, and since $a \in P^{*}$, we get $P=P^{* *} \subseteq(a)^{*}$. To prove the other inclusion, let $b \in(a)^{*}$. Then, $[a, b]=(0) \subseteq P$. Since $P$ is prime, either $a \in P$ or $b \in P$. If $a \in P$, then we have the following:

$$
a \in[a, a] \subseteq\left[P^{*}, P\right]=(0),
$$

which is a contradiction. Therefore, $a \notin P$ and hence $b \in P$. So, $(a)^{*} \subseteq P$ and the equality holds.

(3) Let $I \in i(A)$. Suppose on contrary $I^{*} \vee I^{* *} \neq A$. Then, by applying Zorn's lemma we can find a prime ideal $P$ of $A$ such that $I^{*} \vee I^{* *} \subseteq P$. So, $I^{*} \subseteq P$ and $I^{* *} \subseteq P$, which gives $P^{*} \subseteq I^{*}$. That is, $P^{*} \subseteq P$. By Lemma 3 , we get $P^{*}=(0)$ and hence $P$ is dense, which is a contradiction. Therefore, $I^{*} \vee I^{* *}=A$.

(4) Let $I, J \in i(A)$ such that $[I, J]=(0)$. Then, $J \subseteq I^{*}$. So, $I^{* *} \subseteq J^{*}$, which gives $I^{*} \vee I^{* *} \subseteq I^{*} \vee J^{*}$. It follows from (3) that $I^{*} \vee J^{*}=A$.

Theorem 16. Let $A$ be a c-idempotent algebra. Then, the following conditions are equivalent:

(1) Every proper ideal in $A$ is nondense

(2) Every prime ideal in $A$ is nondense

(3) Every prime ideal is an annihilator ideal

(4) Every ideal is an annihilator ideal

Proof. (1) $\Longrightarrow(2)$ is straight forward and (2) $\Longrightarrow(3)$ is proved in the above theorem. We proceed to show $(3) \Longrightarrow(4)$. Assume (3). Let $I$ be any ideal in $A$. We need to show that $I^{* *} \subseteq I$, suppose not. Then, there is $x \in I^{* *}$ such that $x \notin I$. By the separation axiom, there is a prime ideal $P$ of $A$ such that $I \subseteq P$ and $x \notin P$. This gives $I^{* *} \subseteq P^{* *}$. It follows from our assumption that $I^{* *} \subseteq P$, which is a contradiction. Therefore, $I^{* *} \subseteq I$ and hence it is an annihilator ideal. $(4) \Longrightarrow(1)$ is also straight forward.

Theorem 17. Let $A$ be a c-idempotent algebra. If every prime ideal is nondense, then the following conditions are equivalent: 
(1) Every prime ideal is an annihilator ideal

(2) Every prime ideal is a minimal prime ideal

(3) Every prime ideal is a maximal ideal

Proof. $(1) \Longrightarrow(2)$. Assume (1) and let $P$ be a prime ideal of $A$. Then, $P=P^{* *}$. Let $Q$ be any prime ideal of $A$ such that $Q \subseteq P$. Our aim is to show that $Q=P$, suppose not. Then, there is $x \in P-Q$. For any $a \in P^{*},[a, x]=(0) \subseteq Q$. Since $Q$ is prime and $x \notin Q$, it yields that $a \in Q \subseteq P$. Hence, $a \in P \cap P^{*}=(0)$ so that $P=P^{* *}=A$ which is a contradiction.

$(2) \Longrightarrow(3)$. Assume (2). Let $P$ be a prime ideal of $A$ and $J$ a proper ideal of $A$ such that $P \subseteq J$. By the separation axiom, there exists a prime ideal $Q$ of $A$ such that $J \subseteq Q$ so that $P \subseteq P$. By $P \subseteq P, Q$ is minimal and hence $P=Q$. Therefore, $P=J$ and hence $P$ is maximal.

$(3) \Longrightarrow(1)$. Assume (3) and let $P$ be any prime ideal. Then, it is maximal. It is also clear that $P \subseteq P^{* *}$ and by our assumption $P^{* *}$ is proper. Since $P$ is maximal it yields $P=$ $P^{* *}$ and hence it is an annihilator ideal. The proof ends.

\section{Annihilators in the Sense of Chajda and Halas}

Annihilators in universal algebras have been first studied by Chajda and Halas in the paper [31]. They have used a different approach by introducing the notion of annihilator terms. In this section, we obtain a class of algebras for which the present definition of annihilators is the same as that of annihilators studied by Chajda and Halas. Let us first recall the definition of annihilators proposed by Chajda and Halas in the paper [31].

Definition 15 . Let $\mathscr{K}$ be a class of algebras of the same type $\Omega$ with $0 . \mathscr{K}$ is said to be an annihilator class, whenever it has a so-called annihilator term, i.e., a binary term $q(x, y)$ of type $\Omega$ satisfying the following conditions:

(A1) $q(x, 0)=0=q(0, x)$

(A2) For each $A \in \mathscr{K}$, each ideal term $t(\vec{x}, \vec{y})$ in $\vec{y}$ and every $a_{1}, \ldots, a_{n}, b_{1}, \ldots, b_{m}, c \in A, q\left(b_{i}, c\right)=0$ for all $i=1,2, \ldots, m$ implies $q\left(t\left(a_{1}, \ldots, a_{n}, b_{1}, \ldots\right.\right.$, $\left.\left.b_{m}\right), c\right)=0$.

(A3) For each $A \in \mathscr{K}$ and each $b \in A$, $\langle b\rangle=\{q(x, b): x \in A\}$.

Definition 16. Let $\mathscr{K}$ be an annihilator class and $q(x, y)$ its annihilator term. Let $A \in \mathscr{K}, b \in A$ and $\varnothing \neq M \subseteq A$. The set,

$$
A(b)=\{y \in A: q(y, b)=0\},
$$

will be called the annihilator of the element $b$ in the sense of Chajda and Halas. The set,

$$
A(M)=\cap\{A(b): b \in M\},
$$

will be called the annihilator of $M$ in the sense of Chajda and Halas.
Definition 17. A class $\mathscr{K}$ of algebras is said to satisfy the property $(M)$ if for each $A \in \mathscr{K}$ and each $a, b \in A$; there exists a unique element in $A$ denoted by $p(a, b)$ such that $[a, b]=\langle p(a, b)\rangle$. We call $p(a, b)$ the product of $a$ and $b$.

The variety of groups satisfies the property $(M)$, where $p(x, y)=x^{-1} y^{-1} x y$. The variety of rings has the property $(M)$, where $p(x, y)=x y+y x$. In particular, the class of commutative rings has this property with the product $p(x, y)=x y$. Moreover, the binary term $p(x, y)=x \wedge y$ is a product term in the class of distributive lattices.

Theorem 18. If $\mathscr{K}$ is a class of algebras satisfying the property $(M)$, then it has an annihilator term.

Proof. It is enough to show that $p(x, y)$ is the annihilator term.

(1) Since $[x, 0]=\langle 0\rangle$ for all $x \in A$, it follows that

$$
p(x, 0)=0=p(0, x) \text {. }
$$

(2) Let $t(\vec{x}, \vec{y})$ be any ideal term in $\vec{y}$, $a_{1}, \ldots, a_{n}, b_{1}, \ldots, b_{m}, c \in A$ such that $p\left(b_{i}, c\right)=0$ for all $i=1,2, \ldots, m$, i.e., $\left[b_{i}, c\right]=\{0\}$ for each $i$, which implies that

$$
\bigvee_{i=1}^{m}\left[b_{i}, c\right]=\left[\left\langle\left\{b_{1}, \ldots, b_{m}\right\}\right\rangle, c\right]=\langle 0\rangle,
$$

which gives that $[x, c]=\langle 0\rangle$ for all $x \in\left\langle\left\{b_{1}, \ldots, b_{m}\right\}\right\rangle$. Being $t(\vec{x}, \vec{y})$ an ideal term in $\vec{y}$, we have $t\left(a_{1}, \ldots, a_{n}, b_{1}, \ldots, b_{m}\right) \in$ $\left\langle\left\{b_{1}, \ldots, b_{m}\right\}\right\rangle$ and hence

$$
\left[t\left(a_{1}, \ldots, a_{n}, b_{1}, \ldots, b_{m}\right), c\right]=\langle 0\rangle,
$$

means that $p\left(t\left(a_{1}, \ldots, a_{n}, b_{1}, \ldots, b_{m}\right), c\right)=0$.

(3) Let $a \in A$ and $H=\{p(x, a): x \in A\}$. Our aim is to show that $H=\langle a\rangle$. If $z \in H$, then $z=p(b, a)$ for some $b \in A$, i.e., $\langle z\rangle=[a, b]$. Since $[a, b] \subseteq\langle a\rangle$, it holds that $z \in\langle a\rangle$ and hence $H \subseteq\langle a\rangle$. To prove the other inclusion, let $x \in\langle a\rangle$. Then, there exist an $(n+1)$-ary ideal term $t(\vec{x}, y)$ in $y, a_{1}, \ldots, a_{n} \in A$ such that

$$
x=t\left(a_{1}, \ldots, a_{n}, a\right) .
$$

If $x=0$, then it belongs to $H$. Assume that $x \neq 0$. Now, let us define an $(n+1+1)$-ary term $q(\vec{x}, y, z)$ as follows:

$$
q\left(x_{1}, \ldots, x_{n}, y, z\right)= \begin{cases}0, & \text { if } z=0 \\ t\left(x_{1}, \ldots, x_{n}, y\right), & b \text { otherwise }\end{cases}
$$

Then, $q(\vec{x}, y, z)$ is a commutator term in $y$ and $z$ such that $q\left(a_{1}, \ldots, a_{n}, a, x\right)=x$ which implies that $\langle x\rangle=[a, x]$, i.e., $p(a, x)=x$, so that $x \in H$ and hence $\langle a\rangle \subseteq H$. Therefore, the equality holds and this completes the proof.

The above theorem shows that, for any subset $S$ of $A$ in a class of algebras satisfying the property $(M)$, the annihilator 
$S^{*}$ of $S$ coincides with that of the annihilator $A(S)$ of $S$ in the sense of Chajda and Halas.

\section{Data Availability}

No data were used to support the results of this study.

\section{Conflicts of Interest}

The authors declare that they have no conflicts of interest.

\section{References}

[1] G. Birkenmeier, "Baer rings and quasicontinuous rings have a MDSN," Pacific Journal of Mathematics, vol. 97, no. 2, pp. 283-292, 1981.

[2] G. F. Birkenmeier, "Idempotents and completely semiprime ideals," Communications in Algebra, vol. 11, pp. 567-580, 1983.

[3] G. F. Birkenmeier, "Decompositions of Baer-like rings," Acta Mathematica Hungarica, vol. 59, no. 3-4, pp. 319-326, 1992.

[4] G. F. Birkenmeier, J. Y. Kim, J. K. Park, On Extensions of Quasi-Baer and Principally Quasi-Baer Rings.

[5] W. E. Clark, "Twisted matrix units semigroup algebras," Duke Mathematical Journal, vol. 34, no. 3, pp. 417-423, 1967.

[6] I. Kaplansky, "Rings of operators," Mathematic Lecture Note Series, Benjamin, New York, NY, USA, 1965.

[7] J. W. Fisher, "Von Neumann regular rings versus V-rings," Ring Theory, pp. 101-119, Dekker, New York, NY, USA, 1974.

[8] K. R. Goodearl, "Von Neumann regular rings," Monographs and Studies in Maths, Vol. 4, Pitman, London, UK, 1979.

[9] Y. Utumi, "On continuous regular rings and semisimple self injective rings," Canadian Journal of Mathematics, vol. 12, pp. 597-605, 1960.

[10] R. Y. Chi-Ming, "On Von Neumann regular rings," Mathematical Journal of Okayama University, vol. 14, pp. 151-160, 1980.

[11] R. Y. Chi-Ming, "On regular rings and self-injective rings," Monatshefte für Mathematik, vol. 81, pp. 153-166, 1981.

[12] R. Y. Chi-Ming, "On regular rings and annihilators," Mathematische Nachrichten, vol. 110, no. 1, pp. 137-142, 1983.

[13] A. Atazadeh, M. Sedghi, and R. Naghipour, "On the annihilators and attached primes of top local cohomology modules," Archiv der Mathematik, vol. 102, no. 3, pp. 225-236, 2014.

[14] K. Bahmanpour, "Annihilators of local cohomology modules," Communications in Algebra, vol. 43, no. 6, pp. 25092515, 2015.

[15] K. Bahmanpour, J. A'zami, and G. Ghasemi, "On the annihilators of local cohomology modules," Journal of Algebra, vol. 363 , pp. 8-13, 2012.

[16] S. Rezaei, "On the annihilators of formal local cohomology modules," Hokkaido Mathematical Journal, vol. 48, no. 1, pp. 195-206, 2019.

[17] M. Mandelker, "Relative annihilators in lattices," Duke Mathematical Journal, vol. 37, no. 2, pp. 377-386, 1970.

[18] W. H. Cornish, "Annulets and $\alpha$-ideals in a distributive lattice," Journal of the Australian Mathematical Society, vol. 15, no. 1, pp. 70-77, 1973.

[19] T. P. Speed, "Some remarks on a class of distributive lattices," Journal of the Australian Mathematical Society, vol. 9, no. 3-4, pp. 289-296, 1969.

[20] B. A. Davey, "Some annihilator conditions on distributive lattices," Algebra Universalis, vol. 4, no. 1, pp. 316-322, 1974.
[21] B. A. Davey and J. Nieminen, "Annihilators in modular lattices," Algebra Universalis, vol. 22, no. 2-3, pp. 154-158, 1986.

[22] R. Halaš, "Annihilators and ideals in ordered sets," Czechoslovak Mathematical Journal, vol. 45, pp. 127-134, 1995.

[23] R. Halaš, "Annihilators in BCK-algebras," Czechoslovak Mathematical Journal, vol. 53, no. 128, pp. 1001-1007, 2003.

[24] R. Larsen, "Annihilator ideals in Banach algebras," Monatshefte für Mathematik, vol. 73, no. 5, pp. 400-405, 1969.

[25] R. Halaš and L. Plojhar, "Congruences, ideals and annihilators in standard QBCC-algebras," Central European Journal of Mathematics, vol. 3, pp. 83-97, 2005.

[26] R. Halaš and L. Plojhar, "Annihilators in weakly standard BCC-algebras," International Journal of Mathematics and Mathematical Sciences, vol. 22, pp. 3631-3643, 2005.

[27] M. S. Rao, "On annihilator ideals of C-algebras," Asian-European Journal of Mathematics, vol. 6, pp. 1-11, 2013.

[28] S. K. Vali, P. Sundarayya, and U. M. Swamy, "Annihilators of C-algebras," Southeast Asian Bulletin of Mathematics, vol. 39, pp. 529-538, 2015.

[29] H. P. Gumm and A. Ursini, "Ideals in universal algebras," Algebra Universalis, vol. 19, no. 1, pp. 45-54, 1984.

[30] A. Ursini, "Prime ideals in universal algebras, acta universitatis carolinae," Mathematica et Physica, vol. 25, pp. 75-87, 1984.

[31] I. Chajda and R. Halas, "Annihilators in universal algebra," Contributions to General Algebra, vol. 14, pp. 29-34, 2004.

[32] S. Burris and H. P. Sankappanavor, A Course in Universal Algebra, Springer-Verlag, Berlin, Germany, 1981.

[33] G. Gratzer, General Lattice Theory, Academic Press, New York, NY, USA, 1978.

[34] G. Gratzer, Universal Algebras, Van Nostrand, Princeton, NJ, USA, 1968.

[35] R. Freese and R. McKenzie, "Commutator theory for congruence modular varieties," London Mathematical Society Lecture Note Series, Vol. 125, Cambridge University Press, Cambridge, UK, 1987.

[36] H.-P. Gumm, "An easy way to the commutator in modular varieties," Proceedings of the American Mathematical Society, vol. 80, no. 3, p. 393, 1980.

[37] P. Agliano and A. Ursini, "Ideals and other generalizations of congruence classes," Journal of the Australian Mathematical Society. Series A. Pure Mathematics and Statistics, vol. 53, no. 1, pp. 103-115, 1992.

[38] P. Agliano and A. Ursini, "On subtractive varieties II: general properties," Algebra Universalis, vol. 36, no. 2, pp. 222-259, 1996.

[39] P. Agliano and A. Ursini, "On subtractive varieties III: from ideals to congruences," Algebra Universalis, vol. 37, no. 3, pp. 296-333, 1997.

[40] P. Agliano and A. Ursini, "On subtractive varieties IV: definability of principal ideals," Algebra Universalis, vol. 38, no. 3, pp. 355-389, 1997.

[41] P. Agliano and A. Ursini, "On some ideal basis theorems," Rapporto Matematico N. 182, Universita di Siena, Siena, Italy, 1988.

[42] A. Ursini, "On subtractive varieties, I," Algebra Universalis, vol. 31, no. 2, pp. 204-222, 1994.

[43] R. Padmanabhan, "Equational theory of idempotent algebras," Algebra Universalis, vol. 2, no. 1, pp. 57-61, 1972. 